\title{
THOMAS WILLEBOIRTS BOSSCHAERT: UN NUEVO DIBUJO IDENTIFICADO
}

El dibujo del Ángel mancebo (fig. 1) que retiene nuestra atención figuró con atribución a Abraham Janssens (c. 1675-1632) en el mercado artístico en 1993 ${ }^{1}$. No reconocemos el estilo de este pintor fascinado por la escultura antigua y renacentista, con gran influencia de Miguel Ángel y Caravaggio ${ }^{2}$, que gusta de figuras imponentes y escultóricas, distantes del alargamiento y estilización de la silueta del ángel en este dibujo. En cambio, sí advertimos el modelo de canon alargado y extrema elegancia característico de Thomas Willeboirts Bosschaert (1613/14-1654), uno de los mejores seguidores de Van Dyck, objeto de una reciente monografía ${ }^{3}$ y conocido y citado por preceptistas españoles contemporáneos, como Lázaro Díaz del Valle, que en 1659 señala lo admirable de sus figuras ${ }^{4}$. Fue notoria su conexión con España y su relación con el Conde de Fuensaldaña, uno de sus más importantes mecenas ${ }^{5}$.

El estudio más atento del dibujo nos lleva a asociarlo con el lienzo de gran tamaño que reconoció el profesor Baudoin en la iglesia de San Willibrordus de Berchem, cerca de Amberes, con Dos ángeles sosteniendo el paño de la Verónica, (fig. 2$)^{6}$, siendo el dibujo identificado el ensayo previo para el ángel de la izquierda. Al compararlos, es fácil comprobar que corresponden con exactitud. De otra parte, la grafía del dibujo es la típica del pintor, como puede verse al cotejarlo

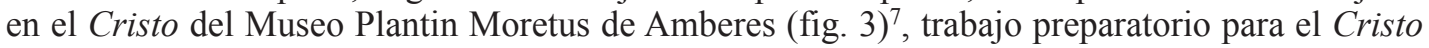

${ }^{1}$ Carboncillo y tiza sobre papel azul, $457 \times 236 \mathrm{~mm}$. Ámsterdam, Christie's, 15-XI-1993, n. ${ }^{\circ} 22$ (como atribuido a Abraham Janssens). Referencia en el RKD de La Haya.

${ }^{2}$ A propósito de Abraham Janssens, véase J. MülLER Hofstede, "Zwei neue Frühwerke von Abraham Janssens", in A. Balis e.a., Florissant. Bijdragen tot de kunstgeschiedenis der Nederlanden (15de-17de eeuw), Liber amicorum Carl Van de Velde, Brusselas, 2005, pp. 271-283; J. VANDER Auwera, Milieu en oeuvre van Abraham Janssen Nuyssen (ca.1571/75-1632), "een seer fameus meester ende schilder in synen levene". Een bijdrage tot de studie van de historieschilderkunst in de Zuidelijke Nederlanden tijdens de eerste helft van de zeventiende eeuw, Gante, Universiteit Gent, tesis doctoral en curso bajo la dirección de prof. Van Damme.

3 A. HeInRICH, Thomas Willeboirts Bosschaert (1613/14-1654). Ein Flämische nachfolger Van Dycks, Turnhout, 2003, 2 vols.

4 "Pintor muy afamado que trabaja admirablemente bien en grandes figuras, es artífice estimado para poder hacer un retrato bastante bien. Su Alteza el Príncipe de Oranje Henrique Federico (...) como también su hijo el príncipe Guillermo y asimismo ha hecho otras para otros monarcas", [Varone Ilustre (M. s., CSIC, fol. 111) Memoria de algunos hombres excelentes que ha habido en España en las artes del dibujo sacadas de un manuscrito de Lázaro Díaz del Valle; cit. M. DíAZ PADRÓN, La pintura flamenca del siglo XVII en España, Universidad Complutense, Tesis Doctoral inédita, Madrid, 1976].

${ }_{5}$ A propósito de la conexión del pintor con España y con el conde de Fuensaldaña, véase M. DíAz PADrón, "Thomas Willeboirts Bosschaert, pintor de Fuensaldaña. Nuevas obras identificadas en Amberes y Estocolmo”, Archivo Español de Arte, 1972, 45, 178, p. 83; M. L. HAIRS, Dans le sillage de Rubens. Les peintres d'historie au XVII' siècle, Lüttich, 1977, p. 243; M. DíAz PADRón, "Dos bocetos de Thomas Willeboirts Bosschaert y de Gerard Seghers atribuidos a Van Dyck del Museo de Picardie y Colección Koetser", Archivo Español de Arte, LIII, 209, 1980, p. 22, n. 10; Idem, Nuevas pinturas de satélites de Rubens inéditas o mal atribuidas, en colecciones españolas y extranjeras: van Lint, van Herp, Th. W. Bosschaert y van Balen, A.E.A. 1988, n. ${ }^{\circ}$ 241, tomo LXI, pp. 1 a 11; Idem, "El boceto de la Inmaculada Concepción de Thomas Willeboirts Bosschaert del Museo de Valladolid en la Johannesburg Art Gallery", Boletín del Seminario de Estudios de Arte y Arqueología de la Universidad de Valladolid (en prensa); Una nueva obra de Thomas WiLlEBOIRTS BossCHAERT, "San Francisco y el ángel músico", Boletín del Seminario de Arte y Arquitectura de Valladolid (en prensa).

${ }^{6}$ Lienzo, ca $215 \times 140 \mathrm{~cm}$. [Véase F. BAudouin, "Ein vermist schilderij van Thomas Willeboirts Bosschaert teruggevonden in de Sint-Willebrorduskerk te Berchem", in Een kompas met vele streken. Studies over Antwerpen, scheepvaart en archivistiek aangeboden aan Dr. Gustaaf Asaert ter gelegenheid van zijn 65ste verjaardag, Amberes, 1994, pp. 31-43; Heinrich 2003, p. 166, Kat n. ${ }^{\circ}$ A13, Abb. 24].

7 Carbón y tiza sobre papel azul, $429 \times 255 \mathrm{~cm}$. Amberes, Museo Plantin Moretus, Stedelijk Prentenkabinet, Inv. N. ${ }^{\circ}$ 129. [Véase M. DíAz PAdrón, "Miscelánea de pintura flamenca del siglo XVII fuera de España", Archivo Español de Arte, 41, 1968, p. 238; HeInRICH, op. cit., 2003, p. 147, Kat. A2a.] 


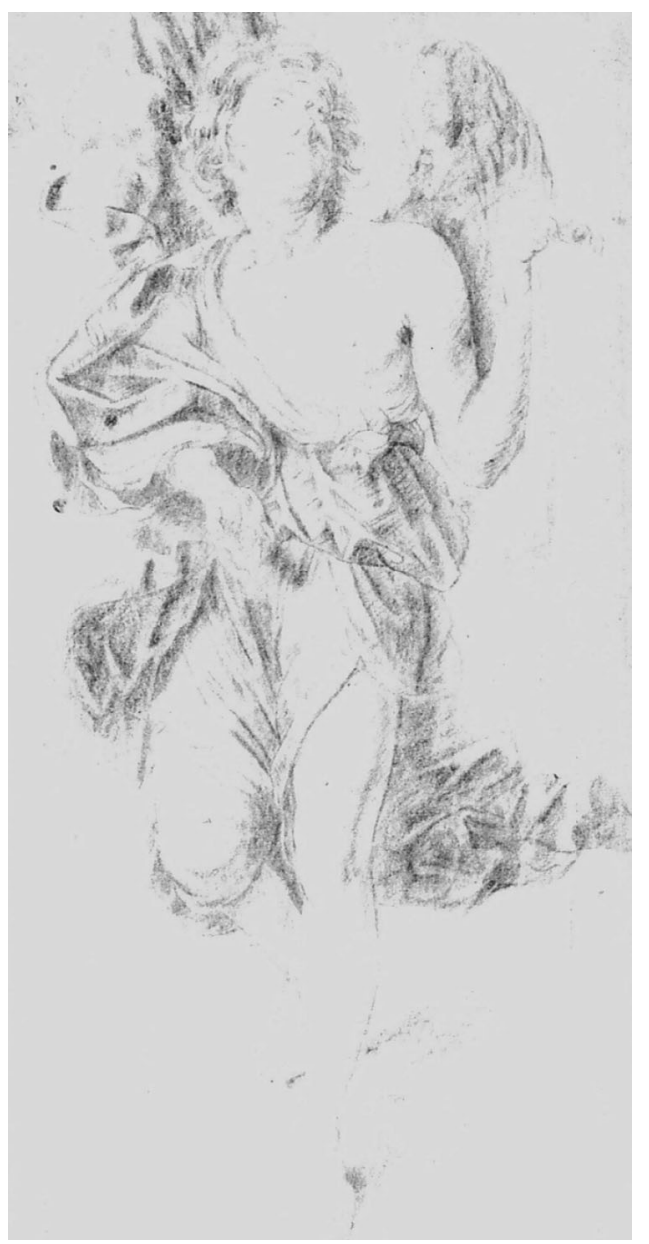

Fig. 1. Thomas Willeboirts Bosschaert. Ángel mancebo sosteniendo el paño de la Verónica. Dibujo, carboncillo y tiza sobre papel. Paradero desconocido.

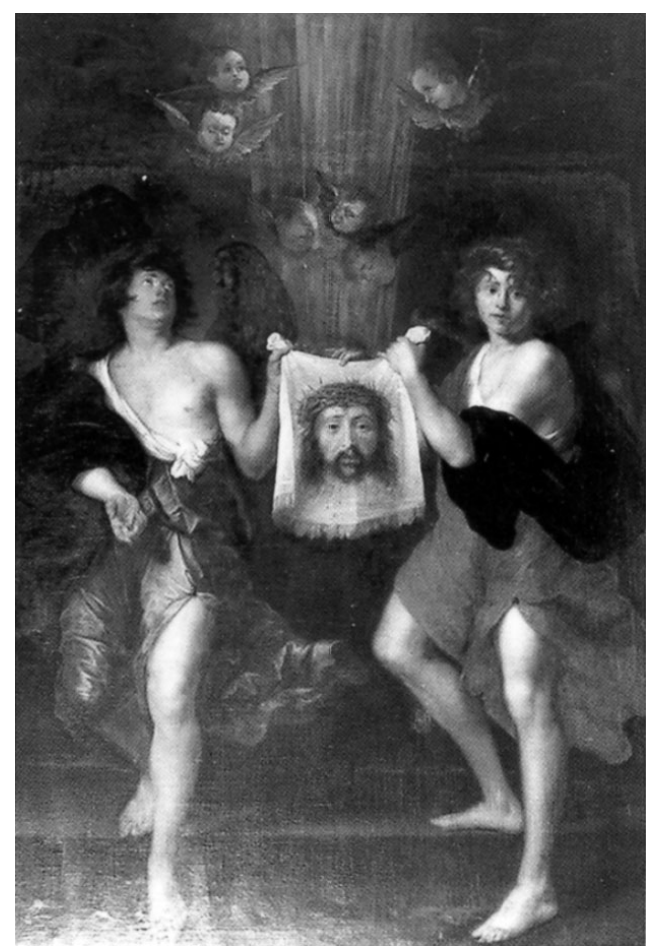

Fig. 2. Thomas Willeboirts Bosschaert.

Dos ángeles con el paño de la Verónica. Berchem (Amberes), Iglesia de St. Willibrordus.

muerto sostenido por dos ángeles de las agustinas de Amberes ${ }^{8}$. En los dos dibujos mencionados trazó los contornos con una línea firme a carboncillo sobre papel azul, marcando las sombras con pequeñas líneas paralelas y las zonas de luz con realces de tiza. Es lógico que una composición de tal ambición y notoria perfección fuera estudiada con detalle y que realizara dibujos previos. Todo indica que Willeboirts tenía por costumbre ensayar sobre papel figuras aisladas para sus mejores composiciones.

Del cuadro definitivo se sabe que, aún en vida del pintor, era propiedad de Anna van Gemert, benefactora del convento de la Anunciación de Amberes, y que fue colocado tras su muerte en 1651 en la capilla de Santa Ana de dicho convento9. Allí fue visto a lo largo del siglo XVIII por

${ }^{8}$ Lienzo, $200 \times 159 \mathrm{~cm}$. Amberes, Claustro de las Agustinas Negras. [Véase DíAz PAdRón, op. cit., 1968, p. 239; HeINRICH, op. cit., 2003, Cat. N. ${ }^{\circ}$ A2, y otras réplicas.]

${ }^{9}$ Figura así en su testamentaría de 1645, donde se señala como heredero a Franciscus de Wolf, sin mención del nombre del autor de la pintura: "Item late ende maeckt aen den heer Franciscus de Wolf bovengenoemt een schilderije resende een schouwstuck daer't Hooft van Onsen Lieven Heer gethoont word door twee encelen, un ter tijt hangende in de beste camer, tot eene gedenckenisse" [Duverger 1991, p. 257, n. ${ }^{\circ} 1376$; cit. HeINRICH, op. cit., 2003, I, p. 166]. En enero de 1651, Ana van Gemert decide cambiar el destino de la pintura, que manda a colocar en el coro del Convento 


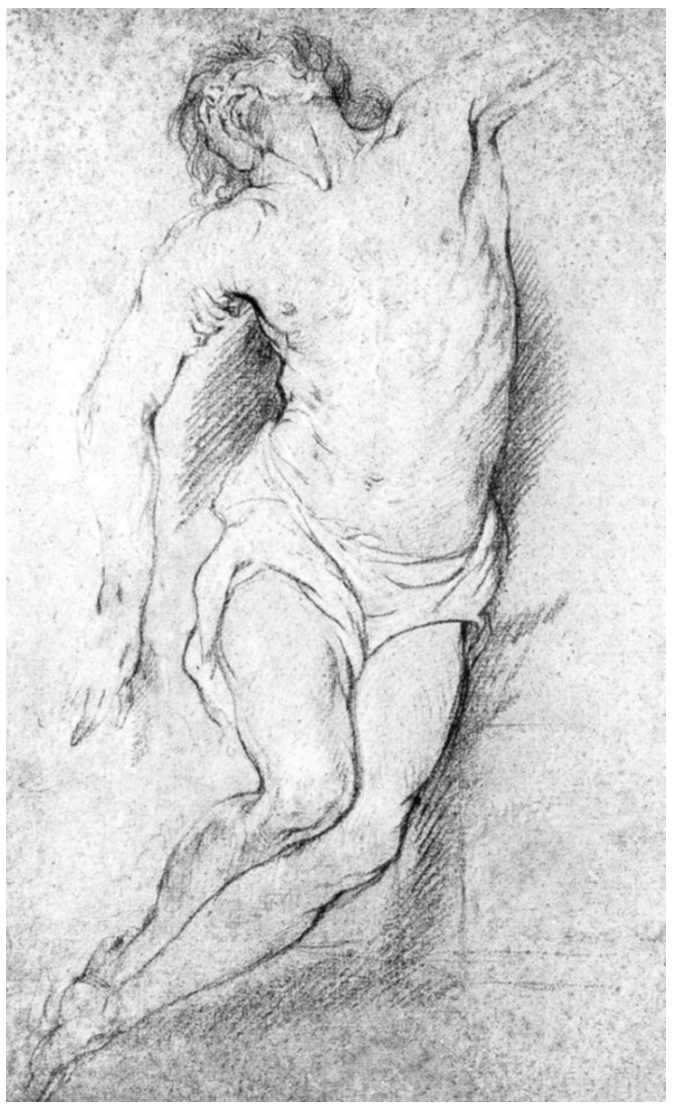

Fig. 3. Thomas Willeboirts Bosschaert.

Cristo muerto. Dibujo, carboncillo y tiza sobre papel azul. Amberes, Museo Plantin-Moretus.

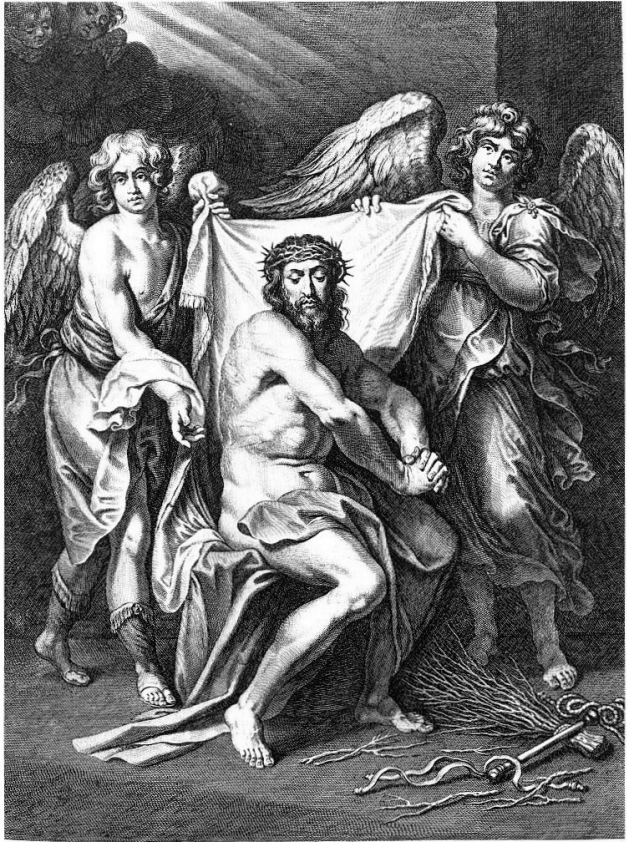

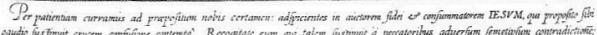

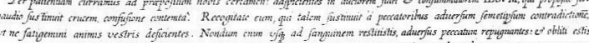

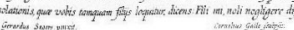

Fig. 4. C. Galle según Gerard Seghers. Cristo consolado por dos ángeles. Grabado.

numerosos viajeros, como Jacob de Witt (1748), el primero en mencionar a Willeboirts como autor y en remarcar su extraordinaria belleza ${ }^{10}$. Descamps, que en un principio habló de la autoría de Willeboirts (1753/54), anota poco después (1769) una posible atribución a Jan Boeckhorst ${ }^{11}$.

de la Anunciación. [Duverger 1992, p. 169, n. ${ }^{\circ} 1671$; cit. HeINRICH, op. cit., 2003, I, p. 166.] Pero a la muerte de la propietaria pocos meses después, el cuadro fue encontrado en el salón principal de su residencia de la Princestraat. [Duverger 1992, p. 204, n. ${ }^{\circ}$ 1696; cit. HeINRICH, op. cit., 2003, I, p. 166.] Posteriormente debió trasladarse a la capilla de Santa Ana de dicho convento, según fue el deseo de su propietaria.

${ }^{10}$ Así escribe De Witt: "In d'eerste capella op den slincker kant is een Doeck van Veronika, opgehouden van twee Engelen, levens groote; door Thomas Willeboirts Bosschaert; extra schoon geschildert; ende Van Dyck soude sigh niet schaaemen van een deerglyck werck aen den daga gebroght te hebben" [Jacobus DE WITT (c. 1748), De kerken van Antwerpen. Met antekeningen door J. de Bosschere en grondplannen, Amberes-La Haya, 1910, pp. 131-122; cit. HEINRICH, op. cit., 2003, p. 166].

11 J. B. DESCAMPS, La vie des peintres flamands, allemands et hollandois, avec des portraits gravés en taille douce, une indication de leurs principaux ouvrages, \& des réflexions sur leurs différaites manières, París, 1753/64, 2, p. 202; J. B. Descamps, Voyage pittoresque de la Flandre et du Brabant, avec des réflexions relativement aux arts \& quelques gravures, París, 1769, p. 200 [cit. HeINRICH, op. cit. 2003, p. 166]. 
El cuadro no escapó a la atención de los entendidos que visitaron el convento. Así, figura mencionado por Mensaert $(1763)^{12}$, Berbie ${ }^{13}$ y Van der Sanden ${ }^{14}$ en sus respectivos escritos, así como por Sir Joshua Reynolds en su Viaje por Flandes y Holanda $(1781)^{15}$. Poco después sería sustraído del convento, y puesto en venta en Bruselas en $1785^{16}$, para ser colocado a mediados del siglo XIX en la iglesia de Santa Willeboirts, donde puede verse en la actualidad. La pintura ostenta acumulación de excrecencias, barnices alterados y un añadido de cinco centímetros en el margen derecho y de cuarenta en la parte superior ${ }^{17}$, donde una mano no muy hábil pintó las tres cabezas de ángeles y los rayos de luz que fluyen hacia el cielo.

Para la composición Willeboirts debió inspirarse en una pintura de Gérard Seghers de la que no se encuentra rastro hoy día pero de cuya existencia en el pasado tenemos prueba por el grabado de Cornelis Galle, con la inscripción "Gerardus Segers pinxit" (fig. 4) ${ }^{18}$. Es lógica esta influencia, pues en 1628, con sólo catorce años, entró Willeboirts en calidad de aprendiz en el taller del ya veterano Seghers ${ }^{19}$. Por estas mismas fechas Van Dyck había vuelto de Italia y, en los años que siguen antes de su partida a Londres en 1632, su influencia sería decisiva en la obra de Willeboirts. De la composición de G. Seghers tomó Willeboirts la figura del ángel que nos ocupa, con actitud de marcha, la mano avanzada con la palma hacia arriba. De Van Dyck, el alargamiento de la silueta y su extrema elegancia. El rostro vuelto hacia atrás, con los ojos tornados hacia arriba es detalle persistente en su obra, que recuerda aquí a la figura de San Juan Evagelista vuelto hacia el águila en el lienzo de los Dos Santos Juanes de la juventud de Van Dyck, perdido en Berlín en la Segunda Guerra Mundial ${ }^{20}$. Puesto que Willeboirts prestó atención a las obras de Van Dyck durante toda su carrera, copiándolo incluso en ocasiones literalmente ${ }^{21}$, es probable que viera la versión de esta pintura que se encontraba en casa de la viuda del muy

12 G. P. Mensaert, Le peintre amateur et curieux, ou description générale des tableaux des plus habiles maîtres, qui font l'ornement des églises, couvents, abbayes, prieurés \& cabinets particuliers dans l'étendue des Pays-Bas Autrichiens, Bruselas, 1793, I, pp. 215-216 [cit. HEINRICH, op. cit., 2003, pp. 166-167].

${ }^{13} \mathrm{G}$. BerBIE, Beschrijvinge der bezonderste werken van de schilder-konste, ende beeldhouwerye, Nu ter tyd zynde in de Kerken, Kloosters, ende opnebare plaetsen der Stad Antwerpen, Amberes, c. 1765, p. 87 [cit. HeINRICH, op. cit., 2003, p. 167].

14 Van der Sanden pensó en un principio que era obra de Van Dyck y, más tarde, de Willeboirts [Jacobus VAN DER SANDEN, Oud Kunst-Toneel van Antwerpen (Manuscrito recogido en 1765-1775), 2, fol. 426, Amberes, Archivo de la ciudad, Privilegekamer, n. ${ }^{\circ}$ 173; cit. HeINRICH, op. cit., 2003, I, p. 166, II, p. 419, nota 117].

15 J. REYNOLDS, "A Journey to Flanders and olland in the year MDCCLXXXI", in The Literary Works of Sir Joshua Reynolds, Londres, 1781, pp. 183-184; cit. HeINRICH, op. cit., 2003, p. 167.

${ }^{16}$ G. Bosschaert, Catalogue d'une collection de tableaux de plusieurs grands maîtres tels que Rubens, Van Dyck. $\&$ plusieurs autres, provenant des maisons religieuses supprimées aux Pays-Bas, dont la vente se fera au Couvent des ci-devant Riches Claires à Bruxelles, en argent de change, Bruselas, 1785, n. 2092.

${ }^{17}$ Se cree que la obra en un principio era de mayores dimensiones. [Véase HEINRICH, op. cit., 2003, p. 167.]

18 Viena, Graphische Sammlung Albertina, Inv. HB 61 (n. ${ }^{\circ}$ 14) [Véase D. BIENECK, Gerard Seghers 1591-1651. Leben und Werk des Antwerpener Historienmalers, 1992, p. 176, n. ${ }^{\circ}$ A55].

19 Vid. P. F. Rombouts y Th. VAn LeRIUS, De Liggeren en andere historische archieven der Antwerpsche Sint Lucasgilde, Amberes-La Haya, 1864-1876, I, p. 661. [Cf. BIENECK, op. cit., 1992, p. 274, doc. 29; HeInRICH, op. cit., 2003, I, p. 19.]

${ }^{20}$ Lienzo, $261 \times 212 \mathrm{~cm}$, firmado: $A^{\text {io }}$ Van Dyck fecit. Se tiene por uno de los tres lienzos ofrecidos al prior de la Abadía de Ter Duinen en Brujas, y vendido en Amberes en 1755 a Francisco el Grande, pasando luego al Palacio de Sanssouci. Fue confiscado por los franceses durante la ocupación, y llevado a París para el Museo Napoleón, devuelto después a los reyes de Prusia, ingresando en Potsdam y luego en la Gemäldegalerie de Berlín, donde se perdió en la Segunda Guerra Mundial. [Véase S. Barnes, N. de Porter, O. Millar y H. Vey, Van Dyck. A complete Catalogue of the Paintings, Londres, 2004, p. 52, n. ${ }^{\circ}$ I.37.] La Academia de Bellas Artes de San Fernando de Madrid conserva el modeletto previo a esta composición. [Tabla, $64 \times 50 \mathrm{~cm}$, véase M. DíAz PADRÓN, Pedro Pablo Rubens (1577-1640), Exposición Homenaje, Madrid, 1977-1978, p. 53, n. $\left.{ }^{\circ} 27.\right]$

${ }^{21}$ Véanse sus copias de Van Dyck en HeInRICH, op. cit., 2003, pp. 311-318.

AEA, LXXXI, 321, ENERO-MARZO 2008, 67-76, ISSN: 0004-0428 
reputado marchante Jan van Haecht, en cuyo inventario aparece descrita en $1627^{22}$. En cualquier caso, no sólo el ángel de la izquierda denota esta influencia de Van Dyck, sino también la idea compositiva general, con los dos ángeles sobre un pedestal y la posición del ángel de la izquierda. Otros paralelismos se pueden observar en el ángel que pintó Van Dyck sosteniendo a San Agustín en éxtasis, pintura que debió causar innegable impacto cuando, hacia 1628, se colocó en el altar izquierdo de la Iglesia de San Agustín de Amberes ${ }^{23}$.

Willeboirts fijó en el dibujo una figura que no modificará en su paso al lienzo definitivo. La precisión va hasta los detalles más mínimos; estudió el atuendo compuesto por túnicas de diferentes colores superpuestas y atadas con un nudo al costado, los pliegues del manto ondeando en el aire, el plumaje de las alas y esbozó incluso el sudario de la Verónica en el margen izquierdo.

Como en la producción de Willeboirts no se advierte una línea evolutiva, es difícil aventurar una fecha para el dibujo que tratamos y la obra definitiva. De una parte, la influencia de los precedentes citados y la proximidad estilística con su maestro y con el Cristo muerto sostenido por dos ángeles del claustro de las Agustinas de Amberes y con su dibujo preparatorio aquí reproducido, conduce a pensar en una fecha temprana; de otra, se hace patente en el lienzo definitivo que el pintor está en plena posesión de su oficio. En cualquier caso, no es posible que exceda el año 1645 , fecha en que el lienzo figura mencionado por primera vez en el testamento de Anna van Gemert, lo que demuestra que de nuestro ángel deriva sin duda el guardián que introdujo en la Huida a Egipto que pintó para Federico Enrique de Oranje en $1647^{24}$, donde repite el rostro vuelto hacia atrás, el gesto elocuente de la mano y la actitud de avance, aunque acusando el movimiento en diagonal.

JAHEL SANZSALAZAR

22 "Item staen in de forie in de winckel van Monsieur de Clerck tot Parys een groot stuck schilderij van Antoni van Dyck geschildert wesende Sint-Jan-Baptist ende Sint-Jan-Evangelist" [E. DUVERGER, Antwerpse kunstinventarissen uit de zeventiende eeuw, Bronnen voor de Kunstgeschiedenis van de Nederlanden, III, p. 54].

${ }^{23}$ Lienzo, $398 \times 227 \mathrm{~cm}$. Amberes, Koninklijk Museum loor Schone Kunsten. [Véase S. BARNES et al., op. cit., 2004, p. 277, n. ${ }^{\circ}$ III.47.]

${ }^{24}$ Lienzo, $218 \times 269 \mathrm{~cm}$. Firmado y fechado: T. WILLEBOIRT 1647 [Postdam, Palacio de Sanssouci, Inv. GK I 5208; véase HeINRICH, op. cit., 2003, cat. N. ${ }^{\circ}$ A42]. 